Proyecciones Journal of Mathematics Vol. 37, No 2, pp. 379-387, June 2018.

Universidad Católica del Norte

Antofagasta - Chile

\title{
On velocity bimagnetic biharmonic particles with energy on Heisenberg space
}

\author{
Talat Körpinar \\ Mus Alparslan University, Turkey \\ Received: August 2017. Accepted: November 2017
}

\begin{abstract}
In this work, we consider velocity bimagnetic biharmonic particle on $3 D$ Heisenberg space in the magnetic field $\mathcal{B}$ and we give the concept of energy. Moreover, we characterize energy conditions of velocity bimagnetic biharmonic particles with Frenet vector field. Therefore, we obtain energy results for bimagnetic particles by Frenet fields in the Heisenberg space.
\end{abstract}

Keywords : Biharmonic particle, Energy, Heisenberg space, Magnetic fields, Symmetries.

M. S. C. 2010 : 53A04, 53A35, 53C80, $83 A 05$. 


\section{Introduction}

In the literary works, certainly one of the significant aim is to acquire the magnetic curves connected to magnetic field B on Riemannian manifold. Therefore, implicit geometrical highlights in the Riemannian manifolds may be utilized to decide the curvature in the magnetic curves. As a result, magnetic curves can easily be determined totally based in any individual manifold. By way of model, [1-7] exhibited that trajectories involved

with magnetic fields described with Riemannian surface region developing a constant Gaussian curvature $\mathrm{K}$ might very conveniently be determined. This excellent kind of study heve been prolonged to specific background spaces. As an illustration, $[8,9]$ acquired specific trajectories connected by way of Kahler magnetic fields by means of presuming background space is a complex type from space. Practically, [10] provided comprehensive recommendation intended for regular flowlines in the contact magnetic field through presuming normal space is simple contact manifold.

Research upon the basic principle has prolonged by means of determining a Killing magnetic fields with the support of Killing vector fields. A variational strategy method on magnetic flows of the Killing magnetic field on 3D was created. Therefore, [11] researched that solution of the formula of Lorentz force can be regarded as Kirchoff elastic rods and the other way round by simply studying magnetic flow on a Killing magnetic field in 3D. After that, [12] handled the magnetic flowlines connected with Killing magnetic fields on the unit sphere in 3D. Finally, [13] explained N and B-magnetic curves as the trajectories of the particular magnetic field and revealed their magnetic flows connected with Killing magnetic field on space. Also some curves and surfaces with energy studied in [14-25].

\section{Heisenberg Space and Frenet Frame}

Heisenberg group is given by with following multipilcation [26].:

$$
(\bar{x}, \bar{y}, \bar{z})(x, y, z)=\left(\bar{x}+x, \bar{y}+y, \bar{z}+z-\frac{1}{2} \bar{x} y+\frac{1}{2} x \bar{y}\right) .
$$

Heisenberg metric $g$ is given by

$$
g=d x^{2}+d y^{2}+(d z-x d y)^{2} .
$$

Heisenberg group's Lie algebra has following orthonormal basis 


$$
\mathbf{d}_{1}=\frac{\partial}{\partial x}, \quad \mathbf{d}_{2}=\frac{\partial}{\partial y}+x \frac{\partial}{\partial z}, \quad \mathbf{d}_{3}=\frac{\partial}{\partial z}
$$

Assume that $\zeta$ be a regular curve in Heisenberg space. Let $\mathbf{e}_{(0)}^{\mu}, \mathbf{e}_{(1)}^{\mu}$, and $\mathbf{e}_{(2)}^{\mu}$ represent the tangent vector, principal normal vector, and secondary normal vector, respectively. For all the curves in Heisenberg space, by transforming the time parameter $t$ into an arc length parameter $s$, the Frenet frame of a curve is defined by

$$
\begin{gathered}
\frac{d \alpha}{d s}=\mathbf{e}_{(0)}^{\mu}, \\
{\left[\begin{array}{c}
\nabla_{\mathbf{e}_{(0)}^{\mu}} \mathbf{e}_{(0)}^{\mu} \\
\nabla_{\mathbf{e}_{(0)}^{\mu}} \mathbf{e}_{(1)}^{\mu} \\
\nabla_{\mathbf{e}_{(0)}^{\mu}}^{\mu} \mathbf{e}_{(2)}^{\mu}
\end{array}\right]=\left[\begin{array}{lll} 
& \kappa & \\
-\kappa & & \tau \\
& -\tau &
\end{array}\right]\left[\begin{array}{c}
\mathbf{e}_{(0)}^{\mu} \\
\mathbf{e}_{(1)}^{\mu} \\
\mathbf{e}_{(2)}^{\mu}
\end{array}\right],}
\end{gathered}
$$

where $\kappa$ and $\tau$ are curvatures of the Frenet frame.

\section{Velocity (Tangent) Bimagnetic Particles of Biharmonic Curves}

Lorentz force $\phi$ connected with a magnetic field $\mathcal{B}$ may easily be calculated simply by

$$
\phi(\mathcal{Y})=\mathcal{B} \times \mathcal{Y}
$$

and consequently [2-8], Lorentz force equation meant for the magnetic particles $\zeta$ may easily be described by way of

$$
\nabla_{\zeta^{\prime}} \zeta^{\prime}=\phi\left(\zeta^{\prime}\right)=\mathcal{B} \times \zeta^{\prime}
$$

$\zeta$ is called a velocity bimagnetic partcle if $\zeta$ is satisfy following 2-Lorentz force equation

$$
\nabla_{\zeta^{\prime}} \nabla_{\zeta^{\prime}} \zeta^{\prime}=\phi\left(\nabla_{\zeta^{\prime}} \zeta^{\prime}\right)=\mathcal{B} \times \nabla_{\zeta^{\prime}} \zeta^{\prime}
$$

Definition 3.1 Energy of a differentiable map $f:(M, \rho) \rightarrow(N, \tilde{h})$ is defined as

$$
\varepsilon(f)=\frac{1}{2} \int_{M} \sum_{a=1}^{n} \tilde{h}\left(d f\left(e_{a}\right), d f\left(e_{a}\right)\right) v
$$


where $\left\{e_{a}\right\}$ is a local basis of the tangent space, $v$ is the canonical volume form $[2,27,28]$.

From above equations Lorentz force of Frenet vector fields of $\zeta$ in Heisenberg space are given by

$$
\begin{aligned}
& \phi\left(\nabla_{\mathbf{e}_{(0)}^{\mu}} \mathbf{e}_{(0)}^{\mu}\right)=\left[\tau \sin ^{2} \mathcal{A} \cos \left[\mathcal{R}_{1} s+\mathcal{R}_{2}\right] \sin \left[\mathcal{R}_{1} s+\mathcal{R}_{2}\right]-\kappa^{2} \cos \mathcal{A}\right] \mathbf{d}_{1} \\
& +\left[-\tau \sin \mathcal{A} \sin \left[\mathcal{R}_{1} s+\mathcal{R}_{2}\right]\left(\mathcal{R}_{1}+\cos \mathcal{A}\right)-\kappa^{2} \sin \mathcal{A} \cos \left[\mathcal{R}_{1} s+\mathcal{R}_{2}\right]\right] \mathbf{d}_{2} \\
& +\left[\tau \mathcal{R}_{1} \sin \mathcal{A} \cos \left[\mathcal{R}_{1} s+\mathcal{R}_{2}\right]-\kappa^{2} \sin \mathcal{A} \sin \left[\mathcal{R}_{1} s+\mathcal{R}_{2}\right]\right] \mathbf{d}_{3}, \\
& \phi\left(\nabla_{\mathbf{e}_{(0)}^{\mu}} \mathbf{e}_{(1)}^{\mu}\right)=\frac{1}{\kappa} \sin ^{2} \mathcal{A} \cos \left[\mathcal{R}_{1} s+\mathcal{R}_{2}\right] \sin \left[\mathcal{R}_{1} s+\mathcal{R}_{2}\right] \mathbf{d}_{1} \\
& -\frac{1}{\kappa} \sin \mathcal{A} \sin \left[\mathcal{R}_{1} s+\mathcal{R}_{2}\right]\left(\mathcal{R}_{1}+\cos \mathcal{A}\right) \mathbf{d}_{2}+\frac{1}{\kappa} \mathcal{R}_{1} \sin \mathcal{A} \cos \left[\mathcal{R}_{1} s+\mathcal{R}_{2}\right] \mathbf{d}_{3}, \\
& \phi\left(\nabla_{\mathbf{e}_{(0)}^{\mu}} \mathbf{e}_{(2)}^{\mu}\right)=\left[-\frac{\tau \rho}{\kappa}\left[\mathcal{R}_{1} \sin ^{2} \mathcal{A} \cos ^{2}\left[\mathcal{R}_{1} s+\mathcal{R}_{2}\right]+\sin ^{2} \mathcal{A} \sin ^{2}\left[\mathcal{R}_{1} s+\mathcal{R}_{2}\right]\left(\mathcal{R}_{1}\right.\right.\right. \\
& +\cos \mathcal{A})]-\kappa \tau \cos \mathcal{A}] \mathbf{d}_{1}+\left[\frac { \tau \rho } { \kappa } \left[\mathcal{R}_{1} \cos \mathcal{A} \sin \mathcal{A} \cos \left[\mathcal{R}_{1} s+\mathcal{R}_{2}\right]\right.\right. \\
& \left.\left.-\sin ^{3} \mathcal{A} \cos \left[\mathcal{R}_{1} s+\mathcal{R}_{2}\right] \sin ^{2}\left[\mathcal{R}_{1} s+\mathcal{R}_{2}\right]\right]-\kappa \tau \sin \mathcal{A} \cos \left[\mathcal{R}_{1} s+\mathcal{R}_{2}\right]\right] \mathbf{d}_{2} \\
& +\left[\frac { \tau \rho } { \kappa } \left[\cos \mathcal{A} \sin \mathcal{A} \sin \left[\mathcal{R}_{1} s+\mathcal{R}_{2}\right]\left(\mathcal{R}_{1}+\cos \mathcal{A}\right)\right.\right. \\
& \left.\left.+\sin ^{3} \mathcal{A} \cos ^{2}\left[\mathcal{R}_{1} s+\mathcal{R}_{2}\right] \sin \left[\mathcal{R}_{1} s+\mathcal{R}_{2}\right]\right]-\kappa \tau \sin \mathcal{A} \sin \left[\mathcal{R}_{1} s+\mathcal{R}_{2}\right]\right] \mathbf{d}_{3} \text {. }
\end{aligned}
$$

\section{Energy of T-Bimagnetic Fields}

In this section, we define energy of velocity bimagnetic particles in the Heisenberg space. Moreover, we give some characterizations and figures of the particle.

Firstly we compute energy of $\left\{\mathbf{e}_{(0)}^{\mu}, \mathbf{e}_{(0)}^{\mu}, \mathbf{e}_{(0)}^{\mu}\right\}$.

$$
\begin{aligned}
& \varepsilon\left(\mathbf{e}_{(0)}^{\mu}\right)=\frac{1}{2} \int_{0}^{s}\left(1+\left[\sin ^{2} \mathcal{A} \cos \left[\mathcal{R}_{1} s+\mathcal{R}_{2}\right] \sin \left[\mathcal{R}_{1} s+\mathcal{R}_{2}\right]\right]^{2}+\left[\operatorname { s i n } \mathcal { A } \operatorname { s i n } [ \mathcal { R } _ { 1 } s + \mathcal { R } _ { 2 } ] \left(\mathcal{R}_{1}\right.\right.\right. \\
& \left.+\cos \mathcal{A})]^{2}+\left[\mathcal{R}_{1} \sin \mathcal{A} \cos \left[\mathcal{R}_{1} s+\mathcal{R}_{2}\right]\right]^{2}\right) d s \\
& \varepsilon\left(\mathbf{e}_{(1)}^{\mu}\right)=\frac{1}{2} \int_{0}^{s}\left(1+\left[\frac { \tau } { \kappa } \left[\mathcal{R}_{1} \sin ^{2} \mathcal{A} \cos ^{2}\left[\mathcal{R}_{1} s+\mathcal{R}_{2}\right]+\sin ^{2} \mathcal{A} \sin ^{2}\left[\mathcal{R}_{1} s+\mathcal{R}\right]\left(\mathcal{R}_{1}\right.\right.\right.\right. \\
& +\cos \mathcal{A})]-\kappa \cos \mathcal{A}]^{2}+\left[-\frac{\tau}{\kappa}\left[\mathcal{R}_{1} \cos \mathcal{A} \sin \mathcal{A} \cos \left[\mathcal{R}_{1} s+\mathcal{R}_{2}\right]\right.\right. \\
& \left.\left.-\sin ^{3} \mathcal{A} \cos \left[\mathcal{R}_{1} s+\mathcal{R}_{2}\right] \sin ^{2}\left[\mathcal{R}_{1} s+\mathcal{R}_{2}\right]\right]-\kappa \sin \mathcal{A} \cos \left[\mathcal{R}_{1} s+\mathcal{R}_{2}\right]\right]^{2} \\
& +\left[-\frac{\tau}{\kappa}\left[\cos \mathcal{A} \sin \mathcal{A} \sin \left[\mathcal{R}_{1} s+\mathcal{R}_{2}\right]\left(\mathcal{R}_{1}+\cos \mathcal{A}\right)\right.\right. \\
& \left.\left.\left.+\sin ^{3} \mathcal{A} \cos ^{2}\left[\mathcal{R}_{1} s+\mathcal{R}_{2}\right] \sin \left[\mathcal{R}_{1} s+\mathcal{R}_{2}\right]\right]-\kappa \sin \mathcal{A} \sin \left[\mathcal{R}_{1} s+\mathcal{R}_{2}\right]\right]^{2}\right) d s, \\
& \varepsilon\left(\mathbf{e}_{(2)}^{\mu}\right)=\frac{1}{2} \int_{0}^{s}\left(1+\left[\frac{\tau}{\kappa} \sin ^{2} \mathcal{A} \cos \left[\mathcal{R}_{1} s+\mathcal{R}_{2}\right] \sin \left[\mathcal{R}_{1} s+\mathcal{R}_{2}\right]\right]^{2}+\left[\frac { \tau } { \kappa } \operatorname { s i n } \mathcal { A } \operatorname { s i n } [ \mathcal { R } _ { 1 } s + \mathcal { R } _ { 2 } ] \left(\mathcal{R}_{1}\right.\right.\right. \\
& \left.+\cos \mathcal{A})]^{2}+\left[\frac{\tau}{\kappa} \mathcal{R}_{1} \sin \mathcal{A} \cos \left[\mathcal{R}_{1} s+\mathcal{R}_{2}\right]\right]^{2}\right) d s .
\end{aligned}
$$




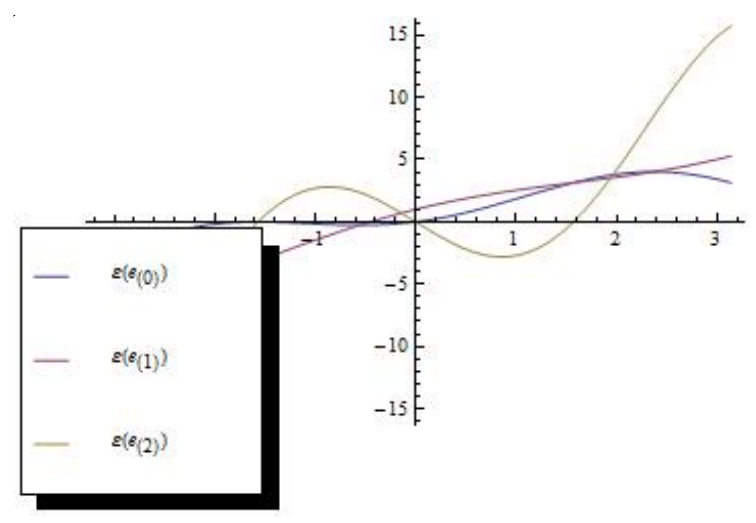

Figure 1. Energy of frenet vectors.

Theorem 4.1 Energy for the particle in the 2-Lorentz force $\phi\left(\nabla_{\mathbf{e}_{(0)}^{\mu}} \mathbf{e}_{(0)}^{\mu}\right)$ is given by

$$
\begin{aligned}
& \varepsilon\left(\phi\left(\nabla_{\mathbf{e}_{(0)}^{\mu}} \mathbf{e}_{(0)}^{\mu}\right)\right)=\frac{1}{2} \int_{0}^{s}\left(1+\left[\tau ^ { 2 } \left[\mathcal{R}_{1} \sin ^{2} \mathcal{A} \cos ^{2}\left[\mathcal{R}_{1} s+\mathcal{R}_{2}\right]+\sin ^{2} \mathcal{A} \sin ^{2}\left[\mathcal{R}_{1} s+\mathcal{R}_{2}\right]\left(\mathcal{R}_{1}\right.\right.\right.\right. \\
& \left.\quad+\cos \mathcal{A})]-\kappa^{2} \sin ^{2} \mathcal{A} \cos \left[\mathcal{R}_{1} s+\mathcal{R}_{2}\right] \sin \left[\mathcal{R}_{1} s+\mathcal{R}_{2}\right]-\kappa^{2} \tau \cos \mathcal{A}\right]^{2} \\
& \quad+\left[\kappa^{2} \sin \mathcal{A} \sin \left[\mathcal{R}_{1} s+\mathcal{R}_{2}\right]\left(\mathcal{R}_{1}+\cos \mathcal{A}\right)-\tau^{2}\left[\mathcal{R}_{1} \cos \mathcal{A} \sin \mathcal{A} \cos \left[\mathcal{R}_{1} s+\mathcal{R}_{2}\right]\right.\right. \\
& \left.\left.\quad-\sin ^{3} \mathcal{A} \cos \left[\mathcal{R}_{1} s+\mathcal{R}_{2}\right] \sin ^{2}\left[\mathcal{R}_{1} s+\mathcal{R}_{2}\right]\right]-\kappa^{2} \tau \sin \mathcal{A} \cos \left[\mathcal{R}_{1} s+\mathcal{R}_{2}\right]\right]^{2} \\
& \quad+\left[-\tau^{2}\left[\cos \mathcal{A} \sin \mathcal{A} \sin \left[\mathcal{R}_{1} s+\mathcal{R}_{2}\right]\left(\mathcal{R}_{1}+\cos \mathcal{A}\right)+\sin ^{3} \mathcal{A} \cos ^{2}\left[\mathcal{R}_{1} s+\mathcal{R}_{2}\right]\right.\right. \\
& \left.\left.\left.\quad \times \sin \left[\mathcal{R}_{1} s+\mathcal{R}_{2}\right]\right]-\kappa^{2} \mathcal{R}_{1} \sin \mathcal{A} \cos \left[\mathcal{R}_{1} s+\mathcal{R}_{2}\right]-\kappa^{2} \tau \sin \mathcal{A} \sin \left[\mathcal{R}_{1} s+\mathcal{R}_{2}\right]\right]^{2}\right) d s
\end{aligned}
$$

Proof. Assume that $\Gamma$ be a moving charged velocity bimagnetic biharmonic curve. Then, we can write

$$
\mathrm{d}(\omega) \circ d(\phi(\mathbf{T}))=d(\omega \circ \phi(\mathbf{T}))=d\left(i d_{C}\right)=i d_{T C} .
$$

Moreoever it is clear that

$$
\mathrm{Q}\left(\phi\left(\mathbf{e}_{(0)}^{\mu}\right)\left(\mathbf{e}_{(\mathbf{0})}^{\mu}\right)\right)=\left[\tau ^ { 2 } \left[\mathcal{R}_{1} \sin ^{2} \mathcal{A} \cos ^{2}\left[\mathcal{R}_{1} s+\mathcal{R}_{2}\right]+\sin ^{2} \mathcal{A} \sin ^{2}\left[\mathcal{R}_{1} s+\mathcal{R}_{2}\right]\left(\mathcal{R}_{1}\right.\right.\right.
$$

$\left.+\cos \mathcal{A})]-\kappa^{2} \sin ^{2} \mathcal{A} \cos \left[\mathcal{R}_{1} s+\mathcal{R}_{2}\right] \sin \left[\mathcal{R}_{1} s+\mathcal{R}_{2}\right]-\kappa^{2} \tau \cos \mathcal{A}\right] \mathbf{d}_{1}$

$+\left[\kappa^{2} \sin \mathcal{A} \sin \left[\mathcal{R}_{1} s+\mathcal{R}_{2}\right]\left(\mathcal{R}_{1}+\cos \mathcal{A}\right)-\tau^{2}\left[\mathcal{R}_{1} \cos \mathcal{A} \sin \mathcal{A} \cos \left[\mathcal{R}_{1} s+\mathcal{R}_{2}\right]\right.\right.$

$\left.\left.-\sin ^{3} \mathcal{A} \cos \left[\mathcal{R}_{1} s+\mathcal{R}_{2}\right] \sin ^{2}\left[\mathcal{R}_{1} s+\mathcal{R}_{2}\right]\right]-\kappa^{2} \tau \sin \mathcal{A} \cos \left[\mathcal{R}_{1} s+\mathcal{R}_{2}\right]\right] \mathbf{d}_{2}$

$+\left[-\tau^{2}\left[\cos \mathcal{A} \sin \mathcal{A} \sin \left[\mathcal{R}_{1} s+\mathcal{R}_{2}\right]\left(\mathcal{R}_{1}+\cos \mathcal{A}\right)+\sin ^{3} \mathcal{A} \cos ^{2}\left[\mathcal{R}_{1} s+\mathcal{R}_{2}\right]\right.\right.$

$\left.\left.\times \sin \left[\mathcal{R}_{1} s+\mathcal{R}_{2}\right]\right]-\kappa^{2} \mathcal{R}_{1} \sin \mathcal{A} \cos \left[\mathcal{R}_{1} s+\mathcal{R}_{2}\right]-\kappa^{2} \tau \sin \mathcal{A} \sin \left[\mathcal{R}_{1} s+\mathcal{R}_{2}\right]\right] \mathbf{d}_{3}$.

Then, it is easy to see that $\varepsilon\left(\phi\left(\nabla_{\mathbf{e}_{(0)}^{\mu}} \mathbf{e}_{(0)}^{\mu}\right)\right)$.

Theorem 4.2 Energy for the particle in the 2-Lorentz force $\phi\left(\nabla_{\mathbf{e}_{(0)}^{\mu}} \mathbf{e}_{(1)}^{\mu}\right)$ is given by 
$\varepsilon\left(\phi\left(\nabla_{\mathbf{e}_{(0)}^{\mu}} \mathbf{e}_{(1)}^{\mu}\right)\right)=\frac{1}{2} \int_{0}^{s}\left(1+\left[\frac{1}{\kappa} \tau\left(-\kappa^{2}-\tau \rho\right)\left[\mathcal{R}_{1} \sin ^{2} \mathcal{A} \cos ^{2}\left[\mathcal{R}_{1} s+\mathcal{R}_{2}\right]\right.\right.\right.$

$\left.+\sin ^{2} \mathcal{A} \sin ^{2}\left[\mathcal{R}_{1} s+\mathcal{R}_{2}\right]\left(\mathcal{R}_{1}+\cos \mathcal{A}\right)\right]-\frac{\tau \rho^{\prime}}{\kappa} \sin ^{2} \mathcal{A} \cos \left[\mathcal{R}_{1} s+\mathcal{R}_{2}\right] \sin \left[\mathcal{R}_{1} s+\mathcal{R}_{2}\right]$

$\left.-\kappa\left(-\kappa^{2}-\tau \rho\right) \cos \mathcal{A}\right]^{2}+\left[-\frac{1}{\kappa} \tau\left(-\kappa^{2}-\tau \rho\right)\left[\mathcal{R}_{1} \cos \mathcal{A} \sin \mathcal{A} \cos \left[\mathcal{R}_{1} s+\mathcal{R}_{2}\right]-\right.\right.$ $\sin ^{3} \mathcal{A} \cos \left[\mathcal{R}_{1} s+\mathcal{R}_{2}\right]$

$\left.\times \sin ^{2}\left[\mathcal{R}_{1} s+\mathcal{R}_{2}\right]\right]+\frac{\tau \rho^{\prime}}{\kappa} \sin \mathcal{A} \sin \left[\mathcal{R}_{1} s+\mathcal{R}_{2}\right]\left(\mathcal{R}_{1}+\cos \mathcal{A}\right)-\kappa\left(-\kappa^{2}-\tau \rho\right)$

$\left.\sin \mathcal{A} \cos \left[\mathcal{R}_{1} s+\mathcal{R}_{2}\right]\right]^{2}+\left[-\frac{1}{\kappa} \tau\left(-\kappa^{2}-\tau \rho\right)\left[\cos \mathcal{A} \sin \mathcal{A} \sin \left[\mathcal{R}_{1} s+\mathcal{R}_{2}\right]\right.\right.$

$\left.\left(\mathcal{R}_{1}+\cos \mathcal{A}\right)+\sin ^{3} \mathcal{A} \cos ^{2}\left[\mathcal{R}_{1} s+\mathcal{R}_{2}\right] \sin \left[\mathcal{R}_{1} s+\mathcal{R}_{2}\right]\right]-\frac{\tau \rho^{\prime}}{\kappa} \mathcal{R}_{1} \sin \mathcal{A} \cos$

$\left.\left.\left[\mathcal{R}_{1} s+\mathcal{R}_{2}\right]-\kappa\left(-\kappa^{2}-\tau \rho\right) \sin \mathcal{A} \sin \left[\mathcal{R}_{1} s+\mathcal{R}_{2}\right]\right]^{2}\right) d s$.

Proof. By using Frenet frame, we obtain

$\phi^{\prime}\left(\nabla_{\mathbf{e}_{(0)}^{\mu}} \mathbf{e}_{(1)}^{\mu}\right)=\left[\frac{1}{\kappa} \tau\left(-\kappa^{2}-\tau \rho\right)\left[\mathcal{R}_{1} \sin ^{2} \mathcal{A} \cos ^{2}\left[\mathcal{R}_{1} s+\mathcal{R}_{2}\right]+\sin ^{2} \mathcal{A}\right.\right.$

$\left.\sin ^{2}\left[\mathcal{R}_{1} s+\mathcal{R}_{2}\right]\left(\mathcal{R}_{1}+\cos \mathcal{A}\right)\right]$

$\left.-\frac{\tau \rho^{\prime}}{\kappa} \sin ^{2} \mathcal{A} \cos \left[\mathcal{R}_{1} s+\mathcal{R}_{2}\right] \sin \left[\mathcal{R}_{1} s+\mathcal{R}_{2}\right]-\kappa\left(-\kappa^{2}-\tau \rho\right) \cos \mathcal{A}\right] \mathbf{d}_{1}$

$+\left[-\frac{1}{\kappa} \tau\left(-\kappa^{2}-\tau \rho\right)\left[\mathcal{R}_{1} \cos \mathcal{A} \sin \mathcal{A} \cos \left[\mathcal{R}_{1} s+\mathcal{R}_{2}\right]-\sin ^{3} \mathcal{A} \cos \left[\mathcal{R}_{1} s+\mathcal{R}_{2}\right]\right.\right.$

$\left.\sin ^{2}\left[\mathcal{R}_{1} s+\mathcal{R}_{2}\right]\right]+\frac{\tau \rho^{\prime}}{\kappa} \sin \mathcal{A} \sin \left[\mathcal{R}_{1} s+\mathcal{R}_{2}\right]\left(\mathcal{R}_{1}+\cos \mathcal{A}\right)-\kappa\left(-\kappa^{2}-\tau \rho\right)$

$\left.\sin \mathcal{A} \cos \left[\mathcal{R}_{1} s+\mathcal{R}_{2}\right]\right] \mathbf{d}_{2}+\left[-\frac{1}{\kappa} \tau\left(-\kappa^{2}-\tau \rho\right)\left[\cos \mathcal{A} \sin \mathcal{A} \sin \left[\mathcal{R}_{1} s+\mathcal{R}_{2}\right]\left(\mathcal{R}_{1}+\right.\right.\right.$ $\left.\cos \mathcal{A})+\sin ^{3} \mathcal{A} \cos ^{2}\left[\mathcal{R}_{1} s+\mathcal{R}_{2}\right] \sin \left[\mathcal{R}_{1} s+\mathcal{R}_{2}\right]\right]-\frac{\tau \rho^{\prime}}{\kappa} \mathcal{R}_{1} \sin \mathcal{A} \cos \left[\mathcal{R}_{1} s+\mathcal{R}_{2}\right]-$ $\left.\kappa\left(-\kappa^{2}-\tau \rho\right) \sin \mathcal{A} \sin \left[\mathcal{R}_{1} s+\mathcal{R}_{2}\right]\right] \mathbf{d}_{3}$.

Theorem 4.3 Energy for the particle in the 2-Lorentz force $\phi\left(\nabla_{\mathbf{e}_{(0)}^{\mu}} \mathbf{e}_{(2)}^{\mu}\right)$ is given by

$\varepsilon\left(\phi\left(\nabla_{\mathbf{e}_{(0)}^{\mu}} \mathbf{e}_{(2)}^{\mu}\right)\right)=\frac{1}{2} \int_{0}^{s}\left(1+\left[\frac{\tau^{2} \rho-\kappa^{2} \tau}{\kappa} \sin ^{2} \mathcal{A} \cos \left[\mathcal{R}_{1} s+\mathcal{R}_{2}\right] \sin \left[\mathcal{R}_{1} s+\mathcal{R}_{2}\right]\right.\right.$

$\left.-\frac{\tau \rho^{\prime}}{\kappa}\left[\mathcal{R}_{1} \sin ^{2} \mathcal{A} \cos ^{2}\left[\mathcal{R}_{1} s+\mathcal{R}_{2}\right]+\sin ^{2} \mathcal{A} \sin ^{2}\left[\mathcal{R}_{1} s+\mathcal{R}_{2}\right]\left(\mathcal{R}_{1}+\cos \mathcal{A}\right)\right]\right]^{2}$

$+\left[-\frac{\tau^{2} \rho-\kappa^{2} \tau}{\kappa} \sin \mathcal{A} \sin \left[\mathcal{R}_{1} s+\mathcal{R}_{2}\right]\left(\mathcal{R}_{1}+\cos \mathcal{A}\right)+\frac{\tau \rho^{\prime}}{\kappa}\left[\mathcal{R}_{1} \cos \mathcal{A} \sin \mathcal{A} \cos \right.\right.$

$\left.\left.\left[\mathcal{R}_{1} s+\mathcal{R}_{2}\right]-\sin ^{3} \mathcal{A} \cos \left[\mathcal{R}_{1} s+\mathcal{R}_{2}\right] \sin ^{2}\left[\mathcal{R}_{1} s+\mathcal{R}_{2}\right]\right]\right]^{2}+\left[\frac{\tau^{2} \rho-\kappa^{2} \tau}{\kappa} \mathcal{R}_{1}\right.$

$\sin \mathcal{A} \cos \left[\mathcal{R}_{1} s+\mathcal{R}_{2}\right]+\frac{\tau \rho^{\prime}}{\kappa}\left[\cos \mathcal{A} \sin \mathcal{A} \sin \left[\mathcal{R}_{1} s+\mathcal{R}_{2}\right]\left(\mathcal{R}_{1}+\cos \mathcal{A}\right)+\right.$ $\left.\left.\left.\sin ^{3} \mathcal{A} \cos ^{2}\left[\mathcal{R}_{1} s+\mathcal{R}_{2}\right] \sin \left[\mathcal{R}_{1} s+\mathcal{R}_{2}\right]\right]\right]^{2}\right) d s$.

Proof. From Theorem 3.4, we get

$\phi^{\prime}\left(\nabla_{\mathbf{e}_{(0)}^{\mu}} \mathbf{e}_{(2)}^{\mu}\right)=\left[\frac{\tau^{2} \rho-\kappa^{2} \tau}{\kappa} \sin ^{2} \mathcal{A} \cos \left[\mathcal{R}_{1} s+\mathcal{R}_{2}\right] \sin \left[\mathcal{R}_{1} s+\mathcal{R}_{2}\right]\right.$

$\left.-\frac{\tau \rho^{\prime}}{\kappa}\left[\mathcal{R}_{1} \sin ^{2} \mathcal{A} \cos ^{2}\left[\mathcal{R}_{1} s+\mathcal{R}_{2}\right]+\sin ^{2} \mathcal{A} \sin ^{2}\left[\mathcal{R}_{1} s+\mathcal{R}_{2}\right]\left(\mathcal{R}_{1}+\cos \mathcal{A}\right)\right]\right] \mathbf{d}_{1}$

$+\left[-\frac{\tau^{2} \rho-\kappa^{2} \tau}{\kappa} \sin \mathcal{A} \sin \left[\mathcal{R}_{1} s+\mathcal{R}_{2}\right]\left(\mathcal{R}_{1}+\cos \mathcal{A}\right)\right.$

$+\frac{\tau \rho^{\prime}}{\kappa}\left[\mathcal{R}_{1} \cos \mathcal{A} \sin \mathcal{A} \cos \left[\mathcal{R}_{\infty} \int+\mathcal{R}_{\in}\right]\right.$

$\left.\left.-\sin ^{\ni} \mathcal{A} \cos \left[\mathcal{R}_{\infty} \int+\mathcal{R}_{\in}\right] \sin ^{\in}\left[\mathcal{R}_{\infty} \int+\mathcal{R}_{\in}\right]\right]\right] \mathbf{d}_{\in}$

$+\left[\frac{\tau^{\epsilon} \rho-\kappa}{\kappa} \tau\right) \mathcal{R}_{\infty} \sin \mathcal{A} \cos \left[\mathcal{R}_{\infty} \int+\mathcal{R}_{\epsilon}\right]$ 
$+\frac{\tau \rho^{\prime}}{\kappa}\left[\cos \mathcal{A} \sin \mathcal{A} \sin \left[\mathcal{R}_{\infty} \int+\mathcal{R}_{\epsilon}\right]\left(\mathcal{R}_{\infty}+\cos \mathcal{A}\right)+\sin ^{\ni} \mathcal{A} \cos ^{\epsilon}\right.$

$\left.\left.\left[\mathcal{R}_{\infty} \int+\mathcal{R}_{\in}\right] \sin \left[\mathcal{R}_{\infty} \int+\mathcal{R}_{\in}\right]\right]\right] \mathbf{d}_{\ni}$ is obtained which gives the result.

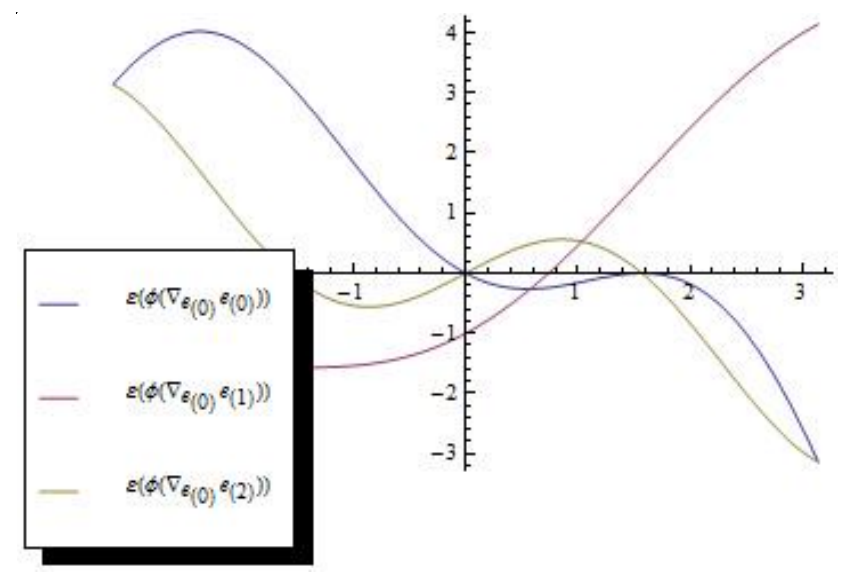

Figure 2. Energy of magnetic frenet vectors.

\section{References}

[1] A. Comtet, On the Landau Hall levels on the hyperbolic plane. Annals of Phys. 173, pp. 185-209, (1987).

[2] S. L. Druta-Romaniuc, M. I. Munteanu, Killing magnetic curves in a Minkowski 3-space. Nonlinear Anal.:Real Word Application. 14 (1), pp. 383-396, (2013).

[3] D. I. Efimov, The magnetic geodesic flows on a homogenous sympletic manifold. Siberian Math. J. 46 (1), pp. 83-93, (2005).

[4] M. I. Munteanu, A. I. Nistor, The classification of Killing magnetic curves in $S^{2} \times R$. J. Geom. Phys. 62 (2), pp. 170-182, (2012). 
[5] S. P. Novikov, The Hamiltonian formalism and a many valued analogue of Morse theory. Russian Math. Surveys 37 (5), pp. 1-56, (1982).

[6] T. Sunada, Magnetic flows on a Riemann surface. Proc. KAIST Math. Workshop, pp. 93-108, (1993).

[7] T. Adachi, Kahler magnetic flow for a manifold of constant holomorphic sectional curvature. Tokyo J. Math. 18 (2), pp. 473-483, (1995).

[8] T. Adachi, Kahler magnetic on a complex projective space. Proc. Japan Acad. Ser. A: Math Sci. 70, pp. 12-13, (1994).

[9] J. L. Cabrerizo, M. Fernandez, J. S. Gomez, On the existence of almost contact structure and the contact magnetic field. Acta Math. Hung. 125 (1-2), pp. 191-199, (2009).

[10] M. Barros, J. L. Cabrerizo, M. Fernandez, A. Romero, Magnetic vortex filament flows. Journal of Math. Phys. 48 (8), (2007), 082904.

[11] J. L. Cabrerizo, Magnetic fields in $2 D$ and $3 D$ sphere. Journal of Nonlinear MAth. Phys. 20 (3), pp. 440-450, (2013).

[12] Z. Bozkurt, İ. Gök, Y. Yayl, F.N. Ekmekci, A new approach for magnetic curves in $3 D$ Riemannian manifolds. Journal of Math. Phys. 55 (2014), 053501.

[13] E. Honig, E. L. Schucking, C. V. Vishveshwara, Motion of charged particles in omogenous electromagnetic fields. Journal of Math. Phys. 15, (1974), doi: 10.1063/1.1666728.

[14] A. Coronel-Escamilla, J. F. Gomez-Angular, E. Alvarado-Mendez, G.V. Guerrero-Ramirez, R. F. Escobar-Jimenez, Fractioanl Dynamics of charged particles in magnetic fields. Int. Journal of Modern Phys. C, 27 (6), (2016).

[15] Z. S. Körpinar, M. Tuz, T. Körpinar, New Electromagnetic Fluids Inextensible Flows of Spacelike Particles and some Wave Solutions in Minkowski Space-time, Int J Theor Phys 55 (1), pp. 8-16, (2016).

[16] T. Körpınar, A New Method for Inextensible Flows of Timelike Curves in Minkowski Space-Time $E_{1}^{4}$, International Journal of Partial Differential Equations, Volume 2014, Article ID 517070, 7 pages. 
[17] T. Körpınar, Bianchi Type-I Cosmological Models for Inextensible Flows of Biharmonic Particles by Using Curvature Tensor Field in Spacetime, Int J Theor Phys 54, pp. 1762-1770, (2015).

[18] T. Körpınar, On the Fermi-Walker Derivative for Inextensible Flows, Zeitschrift für Naturforschung A. 70 (7), pp. 477-482, (2015)

[19] T. Körpinar, B-tubular surfaces in Lorentzian Heisenberg Group H3, Acta Scientiarum. Technology 37 (1), pp. 63-69, (2015).

[20] T. Körpmar, New Characterization for Minimizing Energy of Biharmonic Particles in Heisenberg Spacetime, Int J Phys. 53, pp. 32083218, (2014).

[21] A. Kazan and H.B. Karadağ, 2-Magnetic curves in Euclidean 3-space, Theoretical Mathematics \& Applications, 7 (1), pp. 15-28, (2017).

[22] Z. S. Körpinar, M. Tuz, T. Körpinar, Bianchi Type-I Cosmological Models for Integral Representation Formula and some Solutions in Spacetime, Int J Theor Phys 54 (9), pp. 3195-3202, (2015).

[23] A. Gray, E. Abbena, S. Salamon, Modern Differential Geometry of curves and surfaces with Mathemtica. CRC Press, (1997).

[24] G. Y. Jiang: 2-harmonic maps and their first and second variation formulas, Chinese Ann. Math. Ser. A 7, pp. 389-402, (1986).

[25] S. Rahmani: Metriqus de Lorentz sur les groupes de Lie unimodulaires, de dimension trois, Journal of Geometry and Physics 9 (1992), pp. 295-302, (1992).

[26] C. M. Wood, On the Energy of a Unit Vector Field. Geom. Dedic. 64, pp. 19-330, (1997).

[27] A. Altin, On the energy and Pseduoangle of Frenet Vector Fields in $R_{v}^{n}$. Ukranian Mathematical J. 63 (6), pp. 969-975, (2011).

\section{Talat Körpinar}

Department of Mathematics, Muş Alparslan University, 49250, Muş, Turkey e-mail: talatkorpinar@gmail.com 